\title{
Calidad de vida de mujeres deprimidas en el posparto
}

\author{
G raciela Rojas $C^{1}$, Rosemarie Fritsch $M^{1}$, Jaime Solís $\mathbf{~}^{1}$, \\ Marcos González $A^{2}$, Viviana Guajardo ${ }^{3 a}$, Ricardo Araya $B^{1,4}$. \\ Quality of life of women depressed \\ in the post-partum period
}

Background: Depression is common among women, specially during breeding. Aim: To characterize post-partum depression in a group of women attending a primary health care clinic and its relationship to quality of life. Material and methods: The sample included women meeting criteria for Major Depression, with a child of up to 11 months old. Exclusion criteria included the presence of psychosis, history of mania, alcohol abuse, illegal drug use, high suicide risk, and receiving mental health care in the last three months. Structured interviews used were the Edinburgh postpartum depression scale (EPDS), Mini International Neuropsychiatric Interview (MINI), Medical Outcomes Study Questionnaire (SF-36) and questions about incapacity and health care use. Results: The sample included 159 women with a median age of 27 years (range: 1643 years). Thirty three percent were married, $30.8 \%$ lived in common law marriage, $9.4 \%$ were divorced, and $26.4 \%$ were single. Most (89.3\%) were housewives, $31 \%$ were students, and $6.9 \%$ were employed. The average score on the EPDS was 17 points (S.D. 4.2). The average SF-36 somatic score was 42.7 points (S.D. 8.2), and the emotional score was 30.3 (D.S. 0.3). The relation between the average score on the EPDS and the somatic and emotional scores was statistically significant ( $p=0.000-0.006$ ). Conclusions: Most women were mildly to moderately depressed. Their depression was associated with a marked impairment of activities of daily living. These findings add to the evidence suggesting that depression is associated with marked disability cultures, even when the depression is mild to moderate (Rev Méd Chile 2006; 134: 713-20).

(Key w ords: Depression, postpartum; Primary health care; Quality of life)

Recibido el 9 de marzo, 2005. Aceptado el 8 de noviembre, 2005.

Trabajo financiado por Proyecto FONDECYT \#1040434-04.

${ }_{1}^{1}$ Departamento Psiquiatría y Salud Mental, Hospital Clínico Universidad de Chile. Santiago de Chile. ${ }^{2}$ Instituto Psiquiátrico, Servicio de Salud Metropolitano Norte. ${ }^{3}$ Facultad de Medicina, Universidad de Chile. ${ }^{4}$ Departamento de Psiquiatría, Universidad de Bristol, Reino Unido.

aAlumna de Magíster, Unidad de Epidemiología Clínica.

Correspondencia a: Graciela Rojas C. Avenida La Paz 1003.

Fono: (56-2) 6788601. E mail: grojas@redclinicauchile.cl 
os trastornos depresivos son un problema de _salud pública en todo el mundo, pues son altamente prevalentes y producen grados importantes de discapacidad, estimándose que en el futuro será la segunda causa de años perdidos por discapacidad en el mundo ${ }^{1}$. En Chile ya es, entre las mujeres, la segunda causa de pérdida de años de vida ajustado por discapacidad ${ }^{2}$. La enfermedad depresiva afecta especialmente a las mujeres $\mathrm{y}$, en particular, a las más pobres ${ }^{3-6}$. Se ha descrito mayor incidencia de cuadros depresivos entre mujeres jóvenes en la etapa del ciclo vital que corresponde a la crianza de los hijos ${ }^{4,7-9}$, lo que pudiera explicarse por el aumento creciente de la presión sobre la vida de las familias a través del incremento del trabajo y la desaparición gradual de la familia extendida, restando apoyo a la vida familiar.

La depresión posparto corresponde a una enfermedad depresiva que ocurre en mujeres en un período específico, el puerperio. Se caracteriza por una tendencia al llanto fácil, irritabilidad, por la presencia de sentimientos de culpa y obsesivoscompulsivos, por un rechazo hacia el recién nacido o bien reticencia a asumir su cuidado, por pérdida de la líbido $\mathrm{y}$, en los casos graves, por la presencia de ideación suicida ${ }^{10}$. Se puede detectar a partir de la cuarta semana posterior al alumbramiento ${ }^{11}$, presentándose las mayores tasas de sintomatología depresiva alrededor de la semana 12 posparto ${ }^{12}$. Su prevalencia es tres veces más alta en los países emergentes que en los países desarrollados ${ }^{9,13-15}$. Se han encontrado cifras aproximadas entre 8\% a 30\% de depresión en las mujeres en el período posparto 8,9 . En Chile, se han informado cifras de incidencia de $8,8 \%$ y $9,2 \%$, y de prevalencia puntual a los 2 meses del posparto de $20,5 \%$ y $36,7 \% 8,10,16$.

Los factores de riesgo que han sido descritos para la depresión posparto son variados: edades extremas de las puérperas, el embarazo no deseado, las dificultades conyugales, los problemas con los padres, el nivel socioeconómico, la paridad, la patología gineco-obstétrica de la mujer, el parto por cesárea, el uso de anestesia en el parto, enfermedad del recién nacido y problemas en la lactancia, entre otros ${ }^{11,12,17-21}$. En Chile se ha demostrado que los factores psicosociales juegan un rol preponderante en el desencadenamiento de la depresión posparto. Particular importancia tendrían en las puérperas chilenas la ocurrencia de eventos vitales, la relación con el cónyuge, con la madre y problemas propios del posparto, como dificultades en la lactancia o dar a luz un recién nacido de muy poco peso, así como los antecedentes de depresiones previas ${ }^{22,23}$. Además se ha encontrado una relación inversamente proporcional entre nivel socioeconómico y prevalencia de depresión posparto 24 .

El embarazo y el puerperio representan etapas en la vida de la mujer en que debe enfrentar eventos vitales importantes como los cambios físicos, de roles, etc. Se han realizado estudios en que se han objetivado los cambios experimentados por las mujeres en estas etapas, utilizando mediciones en su calidad de vida ${ }^{25}$. Según la OMS, la calidad de vida es: «a percepción que un individuo tiene de su lugar en la existencia, en el contexto de la cultura y del sistema de valores en los que vive y en relación con sus objetivos, sus expectativas, sus normas, sus inquietudes. Se trata de un concepto muy amplio que está influido de modo complejo por la salud física del sujeto, su estado psicológico, su nivel de independencia, sus relaciones sociales, así como su relación con los elementos esenciales de su entorno». La calidad de vida relacionada con la salud es uno de los parámetros de pronóstico más importantes utilizado en la evolución de una enfermedad y su tratamiento ${ }^{26-30}$, utilizándose como un importante índice de respuesta al momento de evaluar una intervención ${ }^{31,32}$.

También representa una forma de evaluar el impacto de una enfermedad sobre el individuo desde su perspectiva respecto a su experiencia de bienestar-malestar emocional y físico. En nuestro país se han realizado estudios, por los autores ${ }^{33}$, en mujeres con depresión pero no existen estudios con respecto a la DPP.

La evaluación de la calidad de vida en distintas áreas de la medicina no ha sido homogénea, estableciéndose que casi $50 \%$ de los reportes en la literatura se relacionan a enfermedades oncológicas, reumatológicas y músculo-esqueléticas. Sólo $7,5 \%$ se relacionan a enfermedades mentales, a pesar de su gran prevalencia y discapacidad secundaria $^{34}$. La calidad de vida se ha evaluado de diferentes formas, siendo la genérica la más aplicable, por lo que permite comparar diferentes poblaciones de pacientes. 
Variados estudios llevados a cabo en Estados Unidos de Norteamérica y Europa, a nivel poblacional y en la atención primaria, han demostrado el impacto de la depresión en las actividades cotidianas y en la productividad ${ }^{35-37}$ y su correlación con la calidad de vida. Se ha demostrado ampliamente la asociación entre depresión y discapacidad en distintas culturas, sistemas de salud y grados de desarrollo ${ }^{38}$. En Chile los trastornos depresivos se asocian a altos índices de discapacidad $^{2}$. Según la Organización Mundial de la Salud, en el grupo etario entre 15 a 44 años, la depresión unipolar es la enfermedad que más discapacidad produce en ambos sexos ${ }^{1}$.

El objetivo de la presente comunicación es caracterizar el cuadro depresivo que se presenta en el posparto de mujeres consultantes a la atención primaria y su relación con la calidad de vida en dichas mujeres. Estos datos son parte de un ensayo clínico aleatorizado para el tratamiento de la depresión posparto en la atención primaria y esta comunicación corresponde a los datos preliminares de su diagnóstico basal.

\section{MATERIAL Y MÉTODO}

Esta investigación se llevó a cabo en tres consultorios de atención primaria del área norte de Santiago, en cuyas salas de espera se está aplicando la Escala de Depresión Posparto de Edimburgo (EPDS) a todas aquellas madres puérperas, que se encuentren entre los 1 y 11 meses posparto y que no estén embarazadas. Aquellas mujeres que obtienen 10 o más puntos son citadas nuevamente quince días más tarde para una segunda evaluación con el mismo instrumento. Aquéllas que vuelven a presentar 10 o más puntos son evaluadas para la detección de un Episodio Depresivo Mayor y de los siguientes criterios de exclusión: síntomas psicóticos actuales, historia de síntomas maníacos, dependencia o abuso actual de alcohol, consumo actual de drogas ilegales, alto riesgo de suicidio, consulta a psiquiatra o psicólogo en los últimos tres meses $u$ hospitalización por causa psiquiátrica en ese período de tiempo.

Para hacer el diagnóstico de Episodio Depresivo Mayor y para detectar los criterios de exclusión, se utilizó una entrevista estructurada que incluyó el Mini-International Neuropsychiatric
Interview (MINI) y la encuesta del estado de salud SF-36 que ha sido utilizado en estudios internacionales $^{26-30}$ y nacionales ${ }^{33}$.

El MINI es una entrevista diagnóstica estructurada que permite hacer diagnósticos DSM-IV, está disponible en distintos idiomas ${ }^{39}$, entre ellos el español ${ }^{40,41}$. Este instrumento ha sido usado previamente en Chile por miembros de este equipo investigador ${ }^{33}$. El SF-36 es un cuestionario autoaplicado de 36 preguntas que evalúa la calidad de vida, en forma genérica ${ }^{33}$. Constituye el instrumento más utilizado para medir calidad de vida $^{34}$. Consta de ocho dimensiones cuyo puntaje en cada una va de 0 a 100 (siendo 100 la mejor evaluada). A su vez las dimensiones se pueden resumir en 2 índices de salud: índice corporal o físico e índice psíquico o mental ${ }^{33}$.

Estadística. El análisis de los datos se efectuó mediante el programa estadístico SPSS $11.0 \mathrm{y}$ STATA 8.0. Se realizaron pruebas de estadística descriptiva para las variables continuas, tomándose en cuenta su tipo de distribución. Se calcularon los porcentajes de prevalencias para las variables categóricas. Se calcularon índices de las subescalas del SF-36 de acuendo a los algoritmos establecidos. Se realizaron pruebas de correlación y posteriormente se calcularon las curvas de correlación lineal, con sus respectivos parámetros.

\section{RESUlTADOS}

La muestra estuvo constituida por 159 mujeres que acudieron a los consultorios entre la semana 4 y la 44 del período posparto.

Características sociodemográficas. La mediana de edad fue de 27 años (rango entre 16-43). 33,3\% de las mujeres eran casadas, 30,8\% convivientes, $26,4 \%$ solteras y $9,4 \%$ separadas. El $45,9 \%$ de la muestra había cursado educación media completa, 25,8\% educación media incompleta, 10,7\% tenía estudios superiores y 17,6\% tenía educación básica. El 89,3\% de las mujeres desempeñaban labores de dueña de casa, 6,9\% trabajaba, 3,1\% eran estudiantes al momento de la encuesta y $0,6 \%$ se encontraba cesante. Previo al embarazo, 49,7\% era dueña de casa, $27,7 \%$ trabajaba, $22,0 \%$ estudiaba y $0,6 \%$ era cesante. El 69,2\% de las mujeres vivía con la 
Tabla 1. Frecuencia de síntomas depresivos en mujeres deprimidas en el posparto

\begin{tabular}{|lc|}
\hline Síntomas & Frecuencia (\%) \\
\hline Síntomas depresivos & 90,6 \\
Anhedonia & 88,1 \\
Alteraciones apetito/peso & 83,0 \\
Alteraciones del sueño & 79,9 \\
Alteraciones de la psicomotilidad & 77,4 \\
Cansancio/fatiga & 81,8 \\
Ideas de culpa/inutilidad & 65,4 \\
Alteraciones de la concentración/indecisión & 83,0 \\
Ideas de muerte/autoagresión & 35,2 \\
Riesgo suicida leve & 47,8 \\
Riesgo suicida moderado & 11,5 \\
Sin riesgo suicida & 40,8 \\
\hline
\end{tabular}

pareja al momento de la entrevista, $22,0 \%$ con los padres, $6,3 \%$ vivía con otras personas y $2,5 \%$ sola con los hijos. El 61,6\% de las mujeres había tenido un embarazo no planificado y $38,4 \%$ lo había planificado. La muestra tenía un rango de hijos de 1 a 6. La mediana de su periodo posparto fue de 6 meses con un promedio de 5,7 (d.s: 2,9; IC 95\% $5,24-6,16)$.

Características del parto. El 67,9\% de las mujeres habían tenido un parto normal, 28,3\% cesárea y 3,8\% fórceps.

Características clínicas. El puntaje promedio obtenido en el primer EPDS fue de 15 puntos (d.e.: 3,9). El puntaje promedio obtenido en el segundo EPDS fue de 17 puntos (d.e.: 4,2). Entre ambos puntajes existió una correlación de 0,666 $(\mathrm{p}=0,000)$.

Según el MINI, en las últimas dos semanas, $90,6 \%$ de las mujeres estaba deprimida la mayor parte del día, casi todos los días, 88,1\% presentaba anhedonia, 83,0\% tenía alteraciones del apetito y/ o del peso, 79,9\% alteraciones del sueño, 77,4\% alteraciones de la psicomotilidad, $81,8 \%$ se sentía cansada $\mathrm{y} / \mathrm{o}$ fatigada, $65,4 \%$ se sentía culpable 0 inútil, 83,0\% tenía dificultades para concentrarse o tomar decisiones (Tabla 1). El 32,1\% de las mujeres había tenido un episodio depresivo mayor previamente y 33,5\% había recibido tratamien- to con anterioridad. El $64,6 \%$ reconoce antecedentes psiquiátricos familiares, $40,3 \%$ tiene madre, 5\% padre y $14,5 \%$ hermanos con antecedentes psiquiátricos previos.

Calidad de vida. En el índice corporal de la Escala de Calidad de Vida -SF-36-, la muestra obtuvo un puntaje promedio de 42,7 (d.e.: 8,2) y en el índice psíquico un puntaje promedio de 30,3 (d.e.: 10,3). En la Tabla 2 se muestran los puntajes obtenidos por la muestra en las distintas dimensiones del SF-36. La relación entre el puntaje promedio obtenido por la muestra en el EPDS y los índices psíquico y corporal

Tabla 2. Puntajes del cuestionario de salud SF-36 en mujeres deprimidas en el posparto

\begin{tabular}{|lrr|}
\hline Dimensión & \multicolumn{2}{c|}{ Promedio (ds)* } \\
\hline Funcionamiento físico & 69,87 & $(22,59)$ \\
Rol físico & 30,90 & $(33,88)$ \\
Dolor corporal & 47,47 & $(24,43)$ \\
Percepción de salud general & 48,17 & $(19,42)$ \\
Vitalidad & 34,97 & $(19,88)$ \\
Funcionamiento social & 46,28 & $(25,06)$ \\
Rol emocional & 23,84 & $(33,88)$ \\
Salud mental & 40,15 & $(18,11)$ \\
\hline
\end{tabular}

*Los puntajes de las distintas dimensiones van de 0 a 100, siendo 100 el mejor evaluado. 
de la Escala de Calidad de Vida SF-36 es estadísticamente significativa (correlación de pearson $=0,219 \mathrm{y}$ $-0,459$; sig. bilateral $=0,006$ y 0,$000 ; p=0,000$ y $\mathrm{p}=0,000$, respectivamente). Al aplicar un modelo lineal de predicción utilizando el puntaje de la EPDS como variable independiente, y los índices corporal y psíquico como dependientes, se ajustaron en forma estadísticamente significativa. Los parámetros obtenidos para la escala psíquica fueron $\mathrm{b} 0=49,7 \mathrm{y}$ $\mathrm{b} 1=1,1(\mathrm{~F}=41,54 ; \mathrm{p}=0,000)$ y para la física $\mathrm{b} 0=50,1 \mathrm{y}$ $\mathrm{b} 1=0,4(\mathrm{~F}=7,85 ; \mathrm{p}=0,006)$ (Figuras 1 y 2$)$.

\section{DisCUSIÓN}

El cuadro depresivo encontrado en estas mujeres se asoció con grados importantes de discapacidad en las actividades de la vida cotidiana, demostrándose una comelación lineal entre el grado de depresividad y la discapacidad física y psíquica. Esto viene a confirmar la evidencia de que los cuadros depresivos producen grados importantes de discapacidad en distintas culturas, a través de una disminución de la calidad de vida. Uno de los estudios que inició la discusión en tomo a la Calidad de Vida Relacionada a la Salud, fue el Estudio de Pronóstico Médico (Medical Outcomes Study), en el cual los pacientes depresivos fueron comparados con pacientes con diabetes, hipertensión, enfermedad coronaria, angina, artritis, lumbago, problemas pulmonares y trastornos gastrointestinales ${ }^{42}$. Los autores concluyeron que la depresión estuvo asociada con un mayor deterioro físico y social, peor calidad de vida, más días en cama, menos días libres de dolor, mayores costos del tratamiento, y menor percepción del estado de salud; mostrando que la depresión afecta distintos dominios de la salud que son importantes en la consideración del pronóstico y respuesta a tratamiento.

Durante los episodios de depresión, los pacientes experimentaron dificultad en realizar las actividades físicas y se reducen los niveles de energía. La percepción de la salud personal también está afectada, así como sus habilidades para interactuar socialmente, para trabajar y para manejar sus propios hogares ${ }^{33}$. Estos hallazgos se ven corroborados en nuestro estudio, donde las

\section{Curva de Correlación}

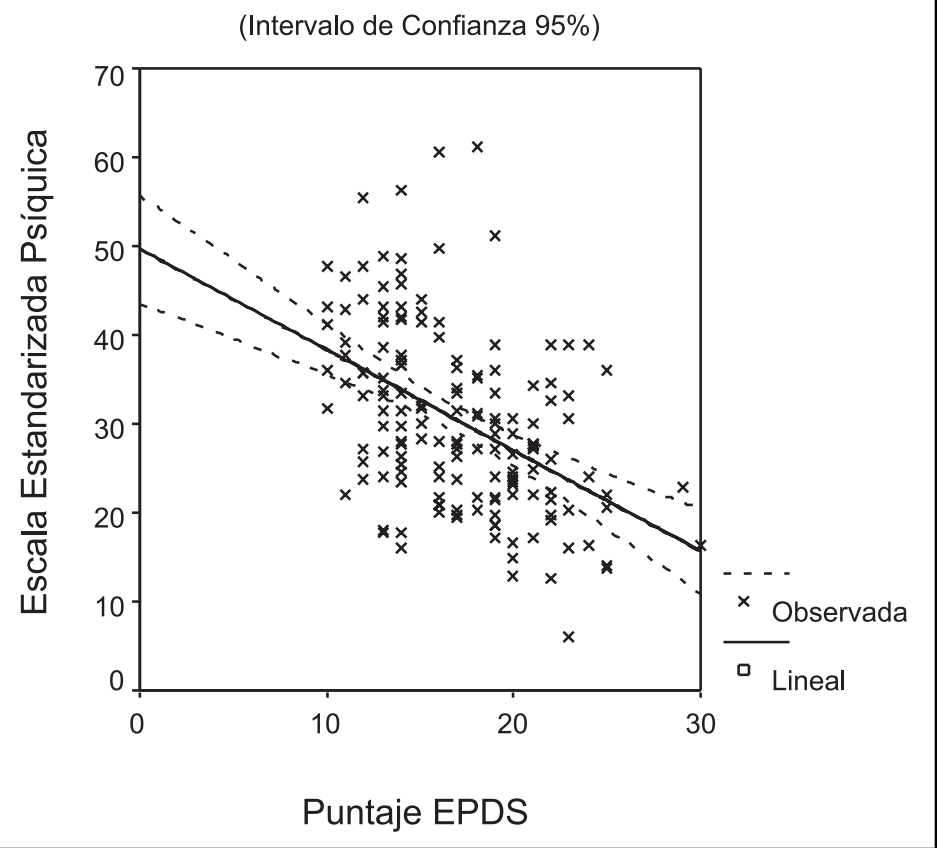

Figura 1. Curva de correlación entre escala estandarizada psíquica y puntaje de EPDS. 
Figura 2. Curva de correlación entre escala estandarizada física y puntaje de EPDS.

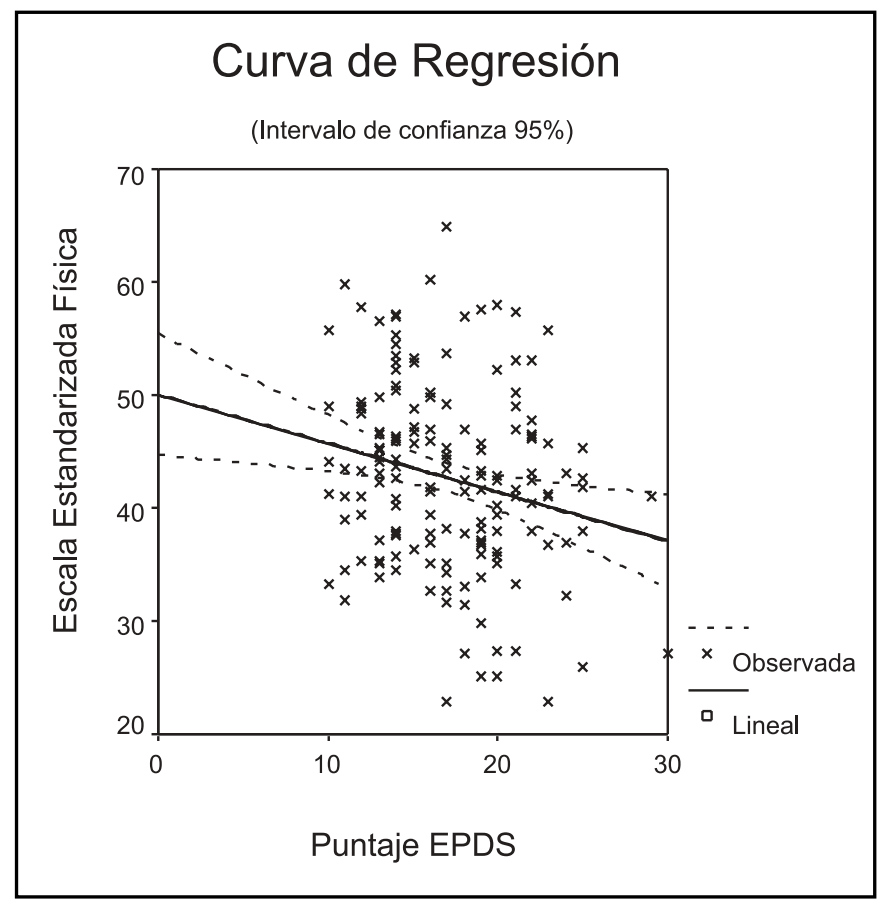

áreas más afectadas fueron el «ol emocional») «el rol físico» y la sitalidad». La mayoría de las mujeres que participaron en este estudio presentaban cuadros depresivos recurrentes lo que podría ser uno de los factores contribuyentes a la mala calidad de vida encontrada en ellas, además de la presencia de un cuadro depresivo actual.

Esta evaluación coincide con resultados internacionales de Dennis y cols que muestran bajos puntajes de las mujeres con EPDS mayor de 12 puntos a las 4 y 8 semanas posparto en las áreas vitalidad, rol emocional y físico del cuestionario SF$36^{43}$. Cabe destacar que las mujeres con DPP están menos discapacitadas que mujeres consultantes en la atención primaria con cuadros depresivos que no se encontraban en el periodo posnatal y que fueron evaluadas con el mismo instrumento en la Región Metropolitana. Sin embargo, si se comparan con mujeres con DPP a nivel internacional éstas tienen una peor percepción de su estado de salud exceptuando en el área de vitalidad ${ }^{44,47}$.

En conclusión la mayoría de estas mujeres presentaron depresión leve a moderada. El cuadro depresivo se asoció con importante discapacidad en las actividades de la vida cotidiana, esto confirma la evidencia que la depresión produce grados signifi- cativos de discapacidad en distintas culturas, incluso en cuadros leves y moderados. Tradicionalmente se ha medido la evolución de la depresión y su respuesta a tratamiento a través de escalas que no consideran la calidad de vida, como la Escala de Depresión de Hamilton, la Escala de Depresión de Montgomery Asberg y la Impresión Clínica Global (CGI), pero éstas no evalúan dominios importantes de la calidad de vida. De aquí la importancia que tiene el uso de cuestionarios que midan la calidad de vida relacionada a la salud de los pacientes depresivos, los que han mostrado ser sensibles en: diferenciar a los pacientes con depresión de los controles $^{45}$, en la detección de síntomas residuales en pacientes que responden a la terapia antidepresi$\mathrm{va}^{46}$, en predecir respuesta a tratamiento ${ }^{47}$ y en detectar diferencias en la respuesta de diferentes antidepresivos ${ }^{48}$.

Los antecedentes de estas mujeres revelan que un porcentaje importante de ellas presentan cuadros depresivos recurrentes y, por lo tanto, debieran ser tratadas en un programa más estructurado de la depresión que permita un seguimiento más estricto, con el objetivo de evitar sus recaídas y la transformación de la enfermedad en un cuadro crónico, lo que a su vez influye en una mala calidad de vida. 


\section{REFERENCIAS}

1. Organización Mundial de la Salud. The World Health Report, 2001. Mental Health: New Understandings, New Hope. Switzerland, World Health Organization 2001.

2. Ministerio DE SAlud. Estudio AVISA, Santiago, MINSAL, 1993.

3. MuRRAY C, LóPEZ A. Altemative projections of mortality and disability by cause 1900-2020: Global Burden of Disease Study. Lancet 1997; 349: 1498-504.

4. Patel V, Araya R, Ludemir A, Todd C, Lima M. Women, proverty and common mental disorders in four restructuring societies. Soc Sci Med 1999; 49: 1461-71.

5. Jenkins R, Lewis $G$, Bebbington P, Brugha T, Farkell M, GILL B ET AL. The national psychiatric morbidity surveys of Great Britain: initial findings from the household survey. Psychol Med 1997; 27: 775-90.

6. Araya R, Rojas G, Fritsch R, Acuña J, Lewis G. Common mental disorders in Santiago, Chile: prevalence and socio-demographic correlates. $\mathrm{Br}$ J Psychiatry 2001; 178: 228-33.

7. Araya R, Rojas G, LEwis G. Comparing sex inequalities in common affective disorders across countries: Great Britain and Chile. Soc Sci Med 2005; 60: 1693-703.

8. JADRESIC E. Trastornos depresivos posparto. En: Psicopatología de la Mujer. Ediciones de la Sociedad de Neurología, Psiquiatría y Neurocirugía de Chile, Chile, 2000. Cap 2.

9. JADRESIC E. Depresión posparto. Rev Chil NeuroPsiquiat 1990; 28: 147-58.

10. Alvarado R, Perucca E, Rojas M, Monardes J, Olea E, Neves E et al. Aspectos gineco-obstétricos en mujeres que desarrollan una depresión posparto. Rev Chil Obstet Ginecol 1993; 58: 239-44.

11. Wisner KL, Peindl KS, Giglotti T, Hanusa BH. Obsessions and compulsions in women with postpartum depression. J Clin Psychiatry 1999; 60: 176-80.

12. Kumar R, Robson KM. A prospective study of emotional disorders in childbearing women. $\mathrm{Br} \mathrm{J}$ Psychiatry 1984; 144: 35-47.

13. Patel V, De Souza N, Rodrigues M. Postnatal depression and infant growth and development in low income countries: a cohort study from Goa, India. Arch Dis Child 2002; 87: 1-4.

14. CoOper P, MurRay L. Postnatal depression. BMJ 1998; 316: 1884-6.
15. Cooper PJ, Tominson M, Swartz L, Woolgar M, MurRay L, Molteno C. Postpartum depression and the mother-infant relationship in a South African peri-urban settlement. Br J Psychiatry 1999; 175: 554-8.

16. Alvarado R, Rojas M, Monardes J, Neves E, Olea E, Perucca E et al. Cuadros depresivos en el posparto y variables asociadas en una cohorte de 125 mujeres embarazadas. Rev Psiquiat 1992; 3: 116876.

17. Kendell RE, Rennie D, Clarke JA, Dean C. The social and obstetric correlates of psychiatric admision in the puerperium. Psychol Med 1981; 11: 341-50.

18. Paykel E, Emms EM, Fletcher J, Rassaby ES. Life events and social support in puerperal depression. Br J Psychiatry 1980; 136: 339-46.

19. Watson J, Ешот SA, Rugg AJ, Brough DI. Psychiatric disorders in pregnancy and the first postnatal year. Br J Psychiatry 1984; 144: 453-62.

20. Appleby L, Forshaw D. Psychiatric disorders of pregnancy and related disorders. In: Postgraduate Psychiatry: Clinical and scientific foundations, Great Britain, 1990; Chap 22.

21. Hopkins J, Marcus M, Campbell SB. Postpartum Depression: A critical review. Psychol Bull 1984; 95: 498-515.

22. JadResic E, JaRA C, ARAya R. La depresión en el embarazo y el puerperio: estudio de factores de riesgo. Acta Psiquiatr Psicol Am Lat 1993; 39: 63-73.

23. Risco L, Jadresic E, Galleguilos T, Garay JL, GonzÁlez M, HasBún J. Depresión posparto: alta frecuencia en puérperas chilenas, detección pre$\mathrm{COz}$, seguimiento y factores de riesgo. Psiquiatría y Salud Integral 2002; 2: 61-6.

24. Jadresic E, Araya R. Prevalencia de depresión posparto y factores asociados en Santiago, Chile. Rev Méd Chile 1995; 123: 694-9.

25. Haas JS, Jackson RA, Fuentes-Afflick E, Stewart AL, Dean ML, BrawarsKy P et al. Changes in the health status of women during and after pregnancy. J Gen Intern Med 2005; 20: 45-51.

26. Smith EM, Gomm SA, Dickens CM. Assessing the independent contribution to quality of life from anxiety and depression in patients with advanced cancer. Palliat Med 2003; 17: 509-13.

27. Jно MY. Study on the correlation between depression and quality of life for Korean women. Nurs Health Sci 2003; 3: 131-7. 
28. Trepanier LL, Rourke SB, Bayoumi aM, Halman MH, KRZYZANowsKi S, Power C. The impact of neuropsychological impairment and depression on health-related quality of life in HIV-infection. J Clin Exp Neuropsychol 2005; 27: 1-15.

29. Goldney RD, Phimps PJ, Fisher LJ, Wilson DH. Diabetes, Depression, and Quality of Life: A population study. Diabetes Care 2004; 27: 106670.

30. Hartmann KE, Ma C, Lamvu GM, Langenberg PW, SteEge JF, KJERULFF KH. Quality of Life and Sexual Function After Hysterectomy in Women With Preoperative Pain and Depression. Obstet Gynecol 2004; 104: 701-9.

31. Otchet F, Carey MS, Adam L. General health and psychological symptom status in pregnancy and the puerperium: what is normal? Obstet Gynecol 1999; 94: 935-41.

32. Symon A, Mackay A, Ruta D. Postnatal quality of life: a pilot study using the Mother-Generated Index. J Adv Nurs 2003; 42: 21-9.

33. Araya R, Rojas G, Fristch R, Gaete J, Rojas M, Simon $G$ ET AL. Treating depression in primary care in low-income women in Santiago. Lancet 2003; 361: 995-1000.

34. Garratt A, Schmidt L, Mackintosh A, Fitzpatrick R. Quality of life measurement: bibliographic study of patient assessed health outcome measures. BMJ 2002; 324: 1417. Review.

35. Broadhead WE, Blazer D, George L, Tse C. Depresion, disability days and day lost from work. JAMA 1990; 264: 2524-8.

36. Spitzer R, Kroenke K, Linzer M, Hahn SR, Wiwiams JBW, DE GRuy FV ET AL. Health related quality of life in primary care patients with mental disorders. JAMA 1995; 274: 1511-7.

37. Von Korfa M, Ormel J, Katon WJ, Lin EHB. Disability and depression among high utilizers of health care. Arch Gen Psychiatry 1992; 49: 91100.

38. Ormel J, KorfF V, Ustun M, Pini B, Korten SA, OLdenhinkel T. Common mental disorders and disability across cultures. Results on psychological problems in general health care. JAMA 1994; 272: $1741-8$.
39. SheEhan DV, Lecrubier Y, Sheehan KH, Amorim P, Janavs J, WeiLer E et al. The Mini-International Neuropsychiatric Interview (MINI): the development and validation of a structured diagnostic psychiatric interview for DSM-IV and ICD-10. J Clin Psychiatry 1998; 59: 22-23, 34-57.

40. Ferrando L, Bobes J, Gibert J, Lecrubier Y. Mini International Neuropsychiatric Interview. En: Bobes J, Bousoño M, González MP, editores. Manejo de los trastornos mentales y del comportamiento en Atención Primaria. 2a ed. Oviedo: Gofer; 1997.

41. Bobes J, González MP, Sáiz PA, Bousoño M, Iglesias C, BASCARÁN MT et AL. La MINI (Mini International Neuropsychiatric Interview): una familia de entrevistas de ayuda diagnóstica en Psiquiatría y Atención Primaria. En: Gutiérrez M, Ezcurra J, González A, Pichot P, editores. Psiquiatría y otras especialidades médicas. Madrid: Grupo Aula Médica; 1998; 401-19.

42. Welis KB, Stewart A, Hays RD, Burnam MA, Rogers $\mathrm{W}$, Daniels $\mathrm{M}$ et al. The functioning and wellbeing of depressed patients: results from the Medical Outcomes Study. JAMA 1989; 262: 914-9.

43. DenNIS CL Influence of depressive symptomatology on maternal health service utilization and general health. Arch Women Ment Health 2004; 7: 183-91.

44. Rojas G, Gaete J, Fritsch R, Rojas M, araya R. Mujer, depresión y calidad de vida en la atención primaria. Rev Psiq Clin 2002; 39: 36-42.

45. Miler IW, Keitner GI, Schatberg AF, Kiein DN, THASE ME, RUSH AJ ET AL. The treatment of chronic depression, part 3: psychosocial funtioning before and after treatment with sertraline or imipramine. J Clin Psychiatry 1998; 59: 608-19.

46. De Lisio G, Maremmani, Perugi G, Cassana GB, Deltito J, Akiskal HS. Impairment of work and leisure in depressed outpatients: a preliminary comunication. J Affect Disor 1986; 25: 213-22.

47. Markowitz JS, Weissman MM, Ouelette R, Lish JD, KIERMAN GL. Quality of life in panic disorder. Arch Gen Psychiatry 1989; 46: 984-92.

48. Kennedy SH, Eisfeld BS, Meyer JH, Bagby RM. Antidepressants in clinical practice: limitations of assessment methods and drugs response. Hum Psychopharmacol Clin Exp 2001; 16: 105-14. 